\title{
On the longest arc relation for $\delta$-subharmonic functions
}

\author{
Alexander Fryntov, John Rossi, and Allen Weitsman
}

\section{Introduction}

A function $u(z)$ defined in the complex plane is called $\delta$-subharmonic if it may be represented as a difference of two subharmonic functions

$$
u(z)=u_{+}(z)-u_{-}(z),
$$

where $u_{+}$and $u_{-}$have no common Riesz mass.

To simplify our further considerations we can assume that $u_{+}(0)=u_{-}(0)=$ 0 . Nevanlinna's characteristics $N(r, u), m(r, u)$ and $T(r, u)$ are defined by

$$
\begin{aligned}
& N(r, u)=\frac{1}{2 \pi} \int_{0}^{2 \pi} u_{-}\left(r e^{i \varphi}\right) d \varphi, \\
& m(r, u)=\frac{1}{2 \pi} \int_{0}^{2 \pi} \max (u, 0)\left(r e^{i \varphi}\right) d \varphi, \\
& T(r, u)=\frac{1}{2 \pi} \int_{0}^{2 \pi} \max \left(u_{+}, u_{-}\right)\left(r e^{i \varphi}\right) d \varphi .
\end{aligned}
$$

Since the representation $u(z)=u_{+}(z)-u_{-}(z)$ is unique up to a harmonic summand, then the characteristic $T(r, u)$ may be defined as

$$
T(r, u)=m(r, u)+N(r, u)
$$

as well.

The order $\rho$ of the function $u(z)$ is defined by

$$
\rho=\limsup _{r \rightarrow \infty} \frac{\log T(r, u)}{\log r} .
$$


Nevanlinna's deficiency of infinity is defined as

$$
\delta=\delta(\infty, u)=\liminf _{r \rightarrow \infty} \frac{m(r, u)}{T(r, u)}=1-\limsup _{r \rightarrow \infty} \frac{N(r, u)}{T(r, u)}
$$

If $f(z)$ is a meromorphic function defined in the whole complex plane, then the function $u(z)=\log |f(z)|$ is $\delta$-subharmonic and the conventional Nevanlinna characteristic of $f(z)$ coincides with that given above for $u$.

The celebrated spread relation of A. Baernstein [3] states that if $f$ is a meromorphic function of finite order $\rho$ and positive Nevanlinna deficiency $\delta=\delta(\infty)$, then

$$
\limsup _{r \rightarrow \infty}|E(r)| \geq \min \left(2 \pi, 4 \rho^{-1} \arcsin \sqrt{\delta / 2}\right) .
$$

Here,

$$
E(r)=\left\{\theta:\left|f\left(r e^{i \theta}\right)\right|>1\right\}
$$

and $|E(r)|$ refers to the angular Lebesgue measure. We note for later reference that (1.1) implies

$$
1-\delta \leq \cos \sigma \delta
$$

where $\sigma$ is half the right hand side of (1.1).

In [4], Baernstein proved that if $f$ is entire and we denote the longest arc in the set $E(r)$ by $L(r)$, then (1.1) is true with $|E(r)|$ replaced by $|L(r)|$ (See also [1]). Later, Weitsman [10] generalized this result to any meromorphic function with $\delta=1$.

We shall prove the analogue of (1.1) with $E(r)$ replaced by $L(r)$ for $\delta$-subharmonic functions. Namely, we prove

Theorem 1.1 Let $U$ be a $\delta$-subharmonic function of order $\rho$. If

$$
L(r)=\text { longest arc of }\{z: U(z)>0\} \cap\{z:|z|=r\},
$$

then

$$
\limsup _{r \rightarrow \infty}|L(r)| \geq \min \left(2 \pi, 4 \rho^{-1} \arcsin \sqrt{\delta / 2}\right) .
$$

We will say that a set $\Omega$ does not contain arcs of opening greater than $2 l$ if for every $r>0$ the intersections

$$
\Omega \cap\{z:|z|=r\}, \quad r>0,
$$


do not contain arcs of angular opening greater that $2 l$.

The analysis of [10] for $\delta=1$, was based on an estimate for the Green's functions of the components of the open set where $\log |f|>0, f$ meromorphic. In [6, Theorem 2], Fryntov proves an estimate involving the circular means of such Green's functions. The proof of Theorem 1.1 will be based on the following modification of Fryntov's result, a modification needed to deal with the slight complication that the set where a $\delta$-subharmonic function is greater than zero need not be open.

Theorem 1.2 Let $\Omega$ be a domain, $M>2$, A be the annulus $\left\{M^{-1}<|z|<\right.$ $M\}$, and $F$ be a countable union of open intervals containing those $r\left(M^{-1}<\right.$ $r<M)$ such that the circle of radius $r$ centered at 0 intersects $\Omega$ with arcs of opening greater than $2 l(0<l<\pi)$. Let $\Omega_{0}$ be the angle $\{z:|\arg z|<l\}$ and $G(z, \xi)$ and $G_{0}(z,|\xi|)$ be the respective Green's functions for $\Omega$ and $\Omega_{0}$ with pole at $\xi \in \Omega$.

If $\varepsilon>0$ is given, then there exists a $\tau(0<\tau<1)$, such that if meas $(F)<$ $\tau$, and $z \in \tilde{A}=\left\{z: \widetilde{M}^{-1}<|z|<\widetilde{M} \mid\right\}$ with $\widetilde{M} / M<\tau$, then

$$
\int_{0}^{2 \pi} G\left(z, R e^{i t}\right) d t \leq \int_{0}^{2 \pi} G_{0}\left(|z|, R e^{i t}\right) d t+\varepsilon \quad\left(\widetilde{M}^{-1}<R<\widetilde{M}\right) .
$$

( Here we assume that $G(z, \xi)$ and $G_{0}(z,|\xi|)$ are zero if either argument is outside $\Omega$ or $\Omega_{0}$ respectively.)

\section{Proof of Theorem 1.2.}

We begin by recalling the method of [6]. For a $\delta$-subharmonic function $u$ defined in an annulus $\left\{z:|z| \in\left(r_{1}, r_{2}\right)\right\}$, let

$$
u(z)=u_{+}(z)-v(z)
$$

be one of its representations as a difference of two subharmonic functions which may have common Riesz mass. Let $\left\{z=r e^{i \theta}: r \in\left(r_{1}, r_{2}\right), \theta \in(0, l)\right\}$ be an annular sector and $u_{l}^{*}$ be defined in the sector by

$$
u_{l}^{*}\left(r e^{i \theta}\right)=\sup \left\{\int_{E} u\left(r e^{i \phi}\right) d \phi: E \in \Gamma(\theta, l)\right\} .
$$


Here $\Gamma(\theta, l)$ is the family of measurable sets of the real axis satisfying the conditions

(a) $|E|=2 \theta$,

(b) $\operatorname{diam}(E) \leq 2 l$,

where (b) means that there exists an arc $I$ such that $|I|=2 l$ and $E \subseteq I$.

As in [6], we apply the notion of $u_{l}^{*}$ to

$$
u(z)=u_{R}(z)=\frac{1}{2 \pi} \int_{-\pi}^{\pi} G\left(z, R e^{i t}\right) d t
$$

where $G$ is the Green's function for $\Omega$ (extended to be 0 outside $\Omega$ ). Thus, in (2.1) we may take

$$
v(z)=\int_{-\pi}^{\pi} \log \left|z-R e^{i t}\right| d t
$$

For the Green's function $G(z)=G(z, \xi)$, and $v(z)$ as in $(2.3)$, we shall then define

$$
T_{l}^{*}\left(r e^{i \theta}, G\right)=u_{l}^{*}\left(r e^{i \theta}\right)+\int_{-\theta}^{\theta} v\left(r e^{i t}\right) d t
$$

which is continuous and subharmonic [6, p 513]. We define $T_{l}^{*}\left(r e^{i \theta}, G_{0}\right)$ similarly.

The proof will rest on a comparison of $T_{l}^{*}(z, G)$ with $T_{l}^{*}\left(z, G_{0}\right)$ in the annular sector

$$
S_{l}=\left\{z=r e^{i \theta}: \theta \in(0, l)\right\} \cap\left\{z: M^{\prime-1}<|z|<M^{\prime}\right\},
$$

where $M^{\prime}\left(\widetilde{M}<M^{\prime}<M\right)$ will be specified later.

Now let $\Psi(z)=T_{l}^{*}(z, G)-T_{l}^{*}\left(z, G_{0}\right)$. Then, as in [6], we observe that $\Psi(z)$ is subharmonic in $S_{l}$ and vanishes on the positive real axis. To estimate $\Psi$ on the inner and outer circular boundary arcs of $S_{\ell}$, let $\widetilde{M}<M^{\prime}<M$. If $\Omega^{*}$ is the circular symmetrization of $\Omega$, and $G_{\Omega^{*}}(z,|\xi|)$ is the Green's function for $\Omega^{*}$ with pole at $|\xi|$, then $[3$, Theorem 5], it follows that

$$
\max _{|z|=r} G(z, \xi) \leq G_{\Omega^{*}}(r,|\xi|)
$$

Using the maximum principle and the fact that the set where $\Omega^{*}$ contains full circles is contained in the set $F$, we deduce that for $\widetilde{M} / M^{\prime}, M^{\prime} / M$ sufficiently small, $\widetilde{M}^{-1}<|\xi|<\widetilde{M}$ and $0 \leq \theta \leq 2 \pi$, 


$$
\begin{gathered}
\max _{\theta} G\left(M^{\prime} e^{i \theta}, \xi\right) \leq \log \left(\frac{M^{\prime 1 / 2}+|\xi|^{1 / 2}}{M^{\prime 1 / 2}-|\xi|^{1 / 2}}\right)+\frac{\varepsilon}{8}<\frac{\varepsilon}{4} \\
\max _{\theta} G\left(M^{\prime}-1 e^{i \theta}, \xi\right) \leq \log \left(\frac{|\xi|^{1 / 2}+M^{\prime-1 / 2}}{|\xi|^{1 / 2}-M^{\prime-1 / 2}}\right)+\frac{\varepsilon}{8}<\frac{\varepsilon}{4} .
\end{gathered}
$$

Thus, by taking $\tau>0$ sufficiently small we may assume

$$
\Psi\left(r e^{i \theta}\right)<\frac{\varepsilon \theta}{2} \quad\left(r=M^{\prime-1}, r=M^{\prime}\right) .
$$

We next estimate $\Psi$ on the $\operatorname{arm} \arg z=\ell$ of $S_{\ell}$. We first observe that there exists a constant $\eta$ such that

$$
\int_{0}^{2 \pi} G\left(z, R e^{i t}\right) d t \leq \eta
$$

The inequality (2.6) follows from the fact that the capacity of the complement of $\Omega$ in the circle centered at the origin of radius $R$ is comparable to $R$.

Let $\sum_{\ell}=S_{\ell} \cup\left\{z: \bar{z} \in S_{\ell}\right\} \cup\left\{z=x+i y: M^{\prime-1}<x<M^{\prime}, y=0\right\}$ and $h(z)$ be the harmonic measure of $\sum_{\ell}$ with respect to the set $\{z: \arg z=$ $\pm \ell,|z| \in F\}$. Then, for $\tau$ sufficiently small we may take

$$
h\left(r e^{i \theta}\right)<\varepsilon / 4 \eta \quad(|\theta| \leq \ell / 2) .
$$

Let $H(z)$ be the harmonic function in $S_{\ell}$ defined by

$$
H\left(r e^{i \theta}\right)=\eta \int_{-\theta}^{\theta} h\left(r e^{i t}\right) d t
$$

Now consider the subharmonic function $\Psi(z)-H(z)$ in $S_{\ell}$. Then $H$ is zero on the real axis, and on $|z|=M^{\prime-1}$ and $M^{\prime}$. We thus consider $\Psi-H$ for points in $\bar{S}_{\ell}$ with $\arg z=\ell$ and $M^{\prime-1}<|z|<M^{\prime}$. For a function $g(z)$ defined in $\bar{S}_{\ell}$ we use the notation

$$
\left.\frac{\partial^{-} g\left(r e^{i \theta}\right)}{\partial \theta}\right|_{\theta=\ell}=\limsup _{\theta \rightarrow \ell^{-}} \frac{g\left(r e^{i \ell}\right)-g\left(r e^{i \theta}\right)}{\ell-\theta} .
$$

The important observation here is that

$$
\left.\frac{\partial^{-} \Psi\left(r e^{i \theta}\right)}{\partial \theta}\right|_{\theta=\ell} \leq \begin{cases}\eta, & r \in F \\ 0, & r \in\left[M^{-1}, M\right] \backslash F .\end{cases}
$$


To verify (2.10), we need only note that for each $\theta$, there is a set $E$ for which the sup in (2.2) is realized (cf.[6, p. 512]) and then apply (2.9) with $g=u_{\ell}^{*}$.

By (2.5),(2.8) and (2.10), we find that the subharmonic function

$$
V(z)=\Psi(z)-H(z)-\frac{\varepsilon \theta}{2}
$$

is 0 on the portion of $\partial S_{\ell}$ on the real axis, is less than or equal to 0 on the portion on $|z|=M^{\prime-1}$ and $|z|=M^{\prime}$, and $\partial^{-} V /\left.\partial \theta\right|_{\theta=\ell}<0$ on the remainder of $\partial S_{\ell}$. Thus, $V(z) \leq 0$ in $S_{\ell}$, or $\Psi\left(r e^{i \theta}\right) \leq H\left(r e^{i \theta}\right)+\frac{\varepsilon \theta}{2}$. Since both sides are 0 when $\theta=0$, the inequality is preserved when one differentiates with respect to $\theta$ and evaluates the derivatives at $\theta=0$. Then using (2.7) and (2.8) we obtain (1.5).

Remark 1.1. With all the notation and hypotheses of Theorem 1.2, let $u(z, R)$ be the harmonic measure of $\Omega \cap\{|z|=R\}$ with respect to $\{|z|=$ $R$ \} and let $u_{0}(z, R)$ be defined similarly with $\Omega_{0}$ in place of $\Omega$. By using the arguments in the proof of Theorem 1.2, we easily obtain the following inequality:

$$
u(z, R) \leq u_{0}(|z|, R)+\varepsilon \quad\left(\widetilde{M}^{-1}<R<\widetilde{M}\right) .
$$

We omit the details.

(We note that the above inequality, with $u_{0}$ multiplied by an absolute constant, can be obtained by using a standard harmonic measure estimate found for example in $[9, \mathrm{p} .112]$, once one realizes that the estimate holds not only for measure but also for longest arc.)

\section{Proof of Theorem 1.1}

Let $U(z)$ be $\delta$-subharmonic function satisfying the conditions of Theorem 1.1, and $\left\{r_{m}\right\}$ be a sequence of Pólya peaks of order $\rho$ of $T(r, U)$. Recall that a sequence $\left\{r_{m}\right\}$ is called a sequence of Pólya peaks of order $\rho$ for $U$ if there exists a positive sequence $\eta_{m} \rightarrow 0$ as $m \rightarrow \infty$, such that

$$
T(r, U) \leq T\left(r_{m}, U\right)\left(r / r_{m}\right)^{\rho}\left(1+\eta_{m}\right), \quad r \in\left[r_{m}^{\prime}, r_{m}^{\prime \prime}\right]
$$

where $r_{m}^{\prime}=\eta_{m} r_{m}, r_{m}^{\prime \prime}=\left(\eta_{m}\right)^{-1} r_{m}$. By [5] such a sequence exists.

If we replace $U$ by $U-1$, then $\left\{r_{m}\right\}$ is again a sequence of Pólya peaks for $T(r, U-1)$, and $\delta$ remains the same. By mollifying $U-1$, we obtain 
continuous $\delta$-subharmonic functions $u=u_{+}-u_{-}=u_{m}$ which, in the Pólya peak annuli $\left\{z: r_{m}^{\prime} \leq|z| \leq r_{m}^{\prime \prime}\right\}$ can be made to approximate $U-1$ (see $[2$, p. 150]) by

$$
|U(z)-1-u(z)|<1 \quad\left(r_{m}^{\prime} \leq|z| \leq r_{m}^{\prime \prime}\right),
$$

outside a countable set of disks, the sum of whose radii is less than any prescribed $\varepsilon_{m}>0$, and $N, m$, and $T$ for $U-1$ are all within $\varepsilon_{m}$ of $N, m$, and $T$ for $u$. For fixed $M$, and $m$ sufficiently large, the open set $\Omega=\Omega_{m}=$ $\{z: u(z)>0\}$ then satisfies the conditions of Theorem 1.2, and meas $(F)$ can be made less than any given $\tau>0$.

By adapting the argument of [8, p. 25] to $\delta$-subharmonic functions, and using the fact that the sums of the diameters of the exceptional disks for (3.1) is arbitrarily small, we may choose sequences $\left\{s_{m}^{(1)}\right\}$ and $\left\{s_{m}^{(2)}\right\}$ so that

$$
\begin{gathered}
s_{m}^{(1)} \in\left(r_{m}^{\prime}, r_{m}\right) ; \quad s_{m}^{(2)} \in\left(r_{m}, r_{m}^{\prime \prime}\right), \\
s_{m}^{(1)} / r_{m}^{\prime} \rightarrow \infty, s_{m}^{(1)} / r_{m} \rightarrow 0, s_{m}^{(2)} / r_{m} \rightarrow \infty, s_{m}^{(2)} / r_{m}^{\prime \prime} \rightarrow 0,
\end{gathered}
$$

and for $i=1,2$

$$
M\left(s_{m}^{(i)}, u\right) \leq K\left(\frac{s_{m}^{(i)}}{r_{m}}\right)^{\rho} T\left(r_{m}, u\right),
$$

where $K$ is independent of $m$ and $M(r, u)$ is the maximum modulus of $u$. Here we have used the Pólya peak inequality along with the $\delta$-subharmonic analogue of the inequality

$$
\frac{1}{r} \int_{1}^{r} M(r, u) d r \leq K(\alpha) T(\alpha r, u),
$$

from [8, p 25], where $\alpha>1$ and $K$ depends only on $\alpha$. We shall estimate $m(r, u)$ by the inequality

$$
\begin{aligned}
m\left(r_{m}, u\right) & =\frac{1}{2 \pi} \int_{0}^{2 \pi} u\left(r_{m} e^{i \theta}\right) d \theta \\
& \leq \iint_{s_{m}^{(1)} \leq|\zeta| \leq s_{m}^{(2)}} \frac{1}{2 \pi} \int_{0}^{2 \pi} G\left(r_{m} e^{i \theta}, \zeta\right) d \nu(\zeta) d \theta \\
& +\sum_{i=1}^{2} \frac{M\left(s_{m}^{(i)}, u\right)}{2 \pi} \int_{0}^{2 \pi} \omega\left(r_{m} e^{i \theta},|z|=s_{m}^{(i)}\right) d \theta \\
& =\mathrm{I}+\mathrm{II},
\end{aligned}
$$


where $\nu$ is the Riesz mass of $u_{-}, G$ is the Green's function for $\Omega$, and $\omega$ is the harmonic measure of $\Omega \cap\left\{s_{m}^{(1)} \leq|z| \leq s_{m}^{(2)}\right\}$ with respect to the circular arcs specified.

Denote the left side of (1.4) by $l$. We may assume that $l<\pi / \rho$; otherwise we are done. This assumption, the fact that meas $(F)$ can be made arbitrarily small, (3.3) with the Pólya peak inequality, and (2.11) show that

$$
\mathrm{II}=o\left(T\left(r_{m}\right)\right), \quad m \rightarrow \infty .
$$

For $s_{m}^{(1)} \leq r,|\zeta| \leq s_{m}^{(2)}$ and $\beta=\pi / l$, we have by Theorem 1.1 that

$$
\frac{1}{2 \pi} \int_{0}^{2 \pi} G\left(r e^{i \theta}, \zeta\right) d \theta \leq \frac{1}{2 \pi} \int_{0}^{2 \pi} G_{0}\left(r e^{i \theta},|\zeta|\right) d \theta+\varepsilon_{m},
$$

where

$$
G_{0}\left(r e^{i \theta}, t\right)=\log \left|\frac{t^{\beta}+\left(r e^{i \theta}\right)^{\beta}}{t^{\beta}-\left(r e^{i \theta}\right)^{\beta}}\right| .
$$

We may take

$$
\varepsilon_{m}<1 / n\left(r_{m}^{\prime \prime}\right),
$$

where $n(t)$ is the $\nu$ measure of the closed disk of radius $t$. Changing the order of integration, integrating by parts twice and using (3.6)-(3.8) (see also [7]; p 126]), we obtain

$$
\begin{aligned}
\mathrm{I} & \leq \frac{1}{2 \pi} \int_{0}^{2 \pi} \int_{s_{m}^{(1)}}^{s_{m}^{(2)}}\left(G_{0}\left(r_{m} e^{i \theta}, t\right)+\varepsilon_{m}\right) d n(t) d \theta \\
& \leq \frac{1}{2 \pi} \int_{s_{m}^{(1)}}^{s_{m}^{(2)}}\left(\frac{2 \beta r^{\beta} t^{\beta}}{t^{2 \beta}+r^{2 \beta}}\right) N(t, U) \frac{d t}{t}-N\left(r_{m}, U\right)+o\left(T\left(r_{m}, U\right)\right)
\end{aligned}
$$

We now use the Pólya peak inequality in (3.9) along with the inequality

$$
N(t, U) \leq(1-\delta+o(1)) T(t, U)
$$

to obtain

$$
\mathrm{I} \leq \frac{2 \beta}{\pi}(1-\delta)\left(\int_{0}^{\infty} \frac{t^{\beta} r^{\beta}}{t^{2 \beta}+r^{2 \beta}}\left(\frac{t}{r_{m}}\right)^{\rho} \frac{d t}{t}+o(1)\right) .
$$

A contour integration of the right side of (3.10), together with (3.4),(3.5) and (1.3) gives that $l$ is at least as large as the right hand side of (1.4). The theorem is proved.

We remark that by using the appropriate Pólya peak sequence, Theorem 1.1 is true with lower order replacing order. 


\section{References}

[1] K. Arima, On maximum modulus of integral functions, J. Math. Soc. Japan, 4 (1952) 62-66.

[2] V.S. Azarin, On the asymptotic behavior of subharmonic functions of finite order, Math. USSR Sbornik (AMS Translations) 36 (1980) 135-154.

[3] A. Baernstein II, A proof of Edrei's spread conjecture, Proc. London Math. Soc. ) 26 (1973) 418-434.

[4] A. Baernstein II, A generalization of the $\cos \pi \rho$ theorem, Trans. Amer. Math. Soc. 193 (1974) 181-197.

[5] A. Edrei, The deficiencies of a meromorphic function of finite lower order, Duke Math. J. 31 (1964) 1-22.

[6] A. Fryntov, Extremal properties of Green functions and A. Weitsman's conjecture, Trans. Amer. Math Soc. 345 (1994) 511-525.

[7] W.Hayman and P. Kennedy, Subharmonic functions vol. 1, Academic Press, (1976).

[8] R. Nevanlinna, Le théorème de Picard-Borel, Chelsea, New York, 2nd ed. (1974).

[9] M. Tsuji, Potential theory in modern function theory, Maruzen Co. Ltd., 1959.

[10] A. Weitsman, Some remarks on the spread of a Nevanlinna deficiency, Inst. Mittag-Leffler, Report no. 7, 1977.

Department of Mathematics, Institute for Low Temperature and Engineering, Kharkov 310164, Ukraine.

Department of Mathematics, Virginia Polytechnic Institute and State University, Blacksburg, Virginia 24061-0123, USA.

Department of Mathematics Purdue University West Lafayette, IN 47907 